\title{
Individualised treatment of proliferative diabetic retinopathy: optimal surgical timing improves long-term outcomes
}

\author{
Stefano Zenoni • Natalia Comi • Piero Fontana
}

Received: 17 December 2009 / Accepted: 28 January 2010/Published online: 10 March 2010

(C) European Association for Predictive, Preventive and Personalised Medicine 2010

\begin{abstract}
Ocular treatment of progressing proliferative diabetic retinopathy is based on retinal laser photocoagulation and pars plana vitrectomy. Improvements in instrumentation and advances in techniques and procedures have increased indications for vitrectomy. These include vitreous haemorrhage preventing laser photocoagulation, severe nonclearing vitreous haemorrhage, subhyaloid and premacular haemorrhage, tractional retinal detachment involving or threatening the macula, combined tractional and rhegmatogenous retinal detachment, progressive fibrovascular proliferation, clinically significant macular oedema and rubeosis iridis. Together with the increasing number of indications, timing for vitrectomy has also changed. The beneficial effect of early vitrectomy for these indications has been clinically shown and supported by several studies. The benefit is evident in case of type I diabetes and when the duration of the diabetes is less than 20 years. Long-term outcomes can therefore be improved when individualised treatment algorithms are applied.
\end{abstract}

Keywords Proliferative diabetic retinopathy - Targeted preventive measures - Individualised treatment algorithms . Improved outcomes

\section{List of abbreviations}

DR Diabetic retinopathy

NPDR Nonpoliferative diabetic retinopathy

PDR Proliferative retinopathy

CSMO Clinically significant macular oedema

\section{S. Zenoni $\cdot$ N. Comi $\cdot$ P. Fontana $(\square)$}

Divisione Oculistica, Azienda Ospedaliera

"Ospedali Riuniti di Bergamo",

Largo Barozzi 1,

24100 Bergamo, Italy

e-mail: pierofontana@hotmail.com
PPV Pars plana vitrectomy

DRVS Diabetic retinopathy vitrectomy study

\section{Introduction}

Diabetic retinopathy (DR) is one of the most common causes of blindness among adults aged between 20 and 74 years and its prevalence continues to increase worldwide [1]. The duration of the disease and the severity of blood glucose fluctuations are major risk factors for the development and progression of the DR [2]. Other risk factors include the age of the patient, the type of diabetes, elevated systemic blood pressure, clotting factors and renal disease. During the first two decades of disease, nearly all patients with type I diabetes and more than $60 \%$ of patients with type II diabetes develop retinopathy [1].

\section{Stages of DR}

Diabetic retinopathy can be classified upon four stages:

1- Mild Nonpoliferative Retinopathy (NPDR). Minimal vascular abnormalities, such as microaneurysms or microhaemorrhages, are present.

2- Moderate Nonproliferative Retinopathy. Presence of microaneurysms, haemorrhages and hard exudates. At this stage closure of some retinal capillaries occurs, leading to hypoxia. Infarction of the nerve fiber layer leads to the formation of cotton-wool spots with associated stasis in axoplasmic flow and retinal oedema.

3- Severe Nonproliferative Retinopathy. As the disease progresses, many vessels are closed thereby depriving 
blood supply to parts of the retina. These ischaemic areas send signals to stimulate proliferation of new blood vessels.

4- Proliferative Retinopathy (PDR). The signals for nourishment, through the induction of angiogenic growth factors, trigger the neovascularisation phenomenon along the retina and the vitreous gel. These new vessels originating from the retina and/or the optic disk can become leaky due to their fragility, leading to preretinal or vitreous haemorrhages (Fig. 1). The majority of the neovascular membranes are adherent to the posterior vitreous cortex. When the posterior hyaloid exerts traction, the edges of the neovascular complex are pulled forwards, resulting in vitreous haemorrhage. The accompanying fibrous tissue can contract resulting in tractional or rhegmatogenous retinal detachment. In the late stage, neovascular glaucoma can result from new vessels growing on the iris and anterior chamber structures.

\section{Breakdown of the blood-retinal barrier as the attribute of DR}

The breakdown of the blood-retinal barrier with increased permeability of the vessels results in leakage of fluid and proteinaceous material, which clinically appears as retinal thickening and exudates. Involvement of the fovea by central retinal thickening and/or lipid deposits (hard exudates) is named clinically significant macular oedema (CSMO) and is the predominant cause of visual loss in DR together with PDR [1].

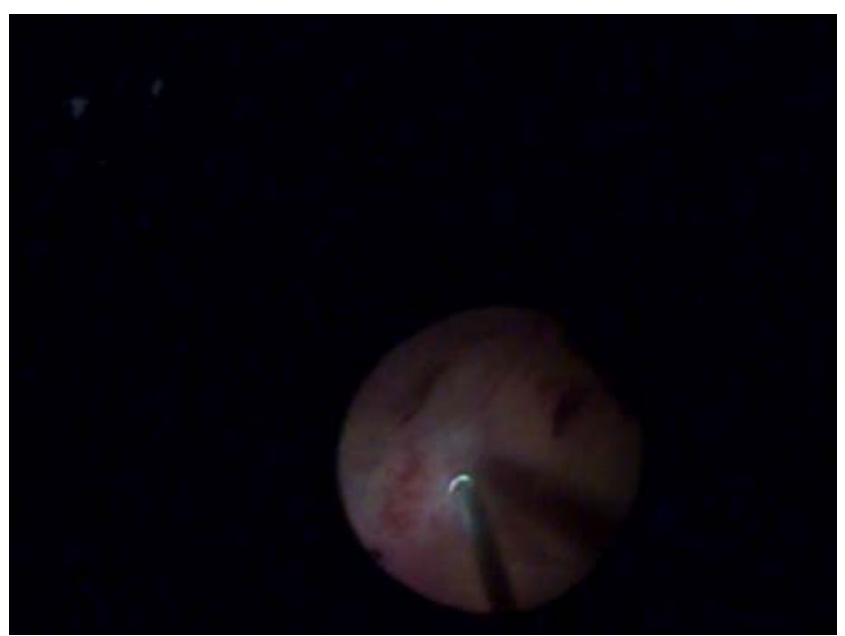

Fig. 1 PPV for vitreous haemorrhage

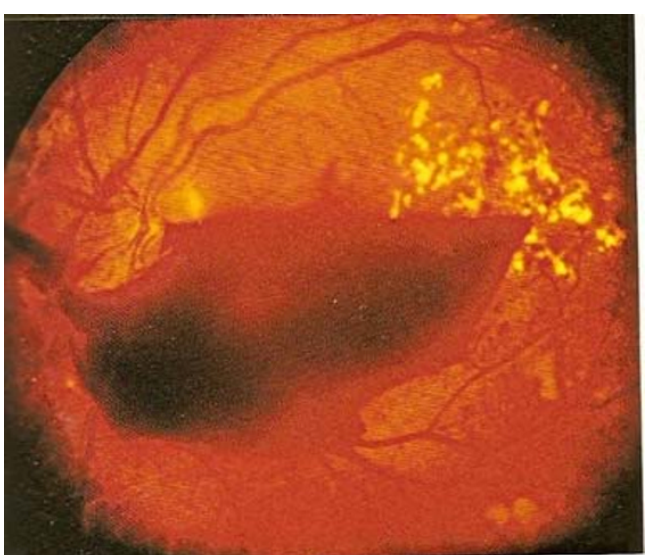

Fig. 2 Vitreous and preretinal haemorrhage from bleeding new vessels

\section{Care and prevention of DR evolution}

At present, glycaemic and blood pressure control remains the standard systemic care to prevent and avoid the evolution of DR. It has been demonstrated that strict glycaemic control significantly reduces the risk of DR development and progression in both types I and II diabetes $[3,4]$. Strict blood pressure control showed a highly significant beneficial effect on the progression of DR and visual loss $[4,5]$.

Several studies found that lipid-lowering drugs are able to reduce hard exudates and inhibit the progression of DR in patients with concomitant dyslipidemia [6,7]. Oral protein kinase $\mathrm{C}$ inhibitors were found to reduce visual loss and the progression of CSMO [8]. Ocular treatment includes laser retinal photocoagulation and vitrectomy. Laser photocoagulation is indicated to ablate ischaemic retinal areas, reducing the induction of neovascularisation. Focal laser treatment at the posterior pole is indicated for CSMO, trying to limit vascular leakage and thereby preventing visual acuity deteriorations.

The current evidence suggests that intravitreal triamcinolone acetonide $[9,10]$ and antivascular endothelial growth factor agents $[11,12]$ are effective adjunctive treatments to laser photocoagulation or vitrectomy.

\section{Pars plana vitrectomy}

The goals of pars plana vitrectomy (PPV) are to remove the vitreous gel, including the posterior hyaloid, and to relieve tractions from the fibrovascular tissue. Although PPV may have intraoperative and postoperative complications, including cataract development, anterior hyaloidal fibrovascular proliferation, fibrovascular ingrowth, recurrent vitreous haemorrhage, retinal detachment and endophthalmitis, it has various indications in the treatment of RD. 
In the recent years further development in vitrectors technology and other instrumentations (e.g., small gauge vitrectomy systems, endolaser and optic fibers, the use of perfluorocarbonades and tamponating agents) together with the refinements of techniques have improved the safety and efficacy of PPV thereby expanding its indications in the treatment of DR. PPV was once reserved only for massive persisting vitreous haemorrhage or retinal detachment.

At present, the most common indications for PPV include: vitreous haemorrhage preventing laser photocoagulation, severe nonclearing vitreous haemorrhage (Fig. 2), subhyaloid and premacular haemorrhage, tractional retinal detachment involving or threatening the macula, combined tractional and rhegmatogenous retinal detachment, progressive fibrovascular proliferation, CSMO and rubeosis iridis [13].

\section{Timing of PPV}

The improved safety of PPV has not only increased the indications to perform it, but also changed its timing. Vitreous haemorrhage tends to spontaneously resolve in about $20 \%$ of patients and in $13 \%$ of type I diabetes; nonetheless, recurrences are frequently observed. Beyond severity and duration, the open issue has therefore become a timing indication.

In the Diabetic Retinopathy Vitrectomy Study (DRVS) 616 eyes with recent severe vitreous haemorrhage reducing visual acuity to $5 / 200$ or less for at least 1 month were randomly assigned to either early vitrectomy or deferral of vitrectomy for 1 year [14]. Patients were classified as having type I or type II diabetes and the duration of diabetes. The 4-year results indicate that visual acuity recovery was more frequent with early vitrectomy in type I diabetes patients compared with deferred vitrectomy. This recovery was greater when the duration of diabetes was less than 20 years. No significant final clinical advantage was appreciated if early vitrectomy was performed in the mixed or type II diabetes. This difference in treatment effects by diabetes type and duration was generally statistically significant for the $10 / 20$ or better visual acuity outcome. This result was achieved in $25 \%$ of the early-treatment patients and in $15 \%$ of the deferral group. Other characteristics as the type of vitreous haemorrhage, its duration, the history of retinal photocoagulation and the initial visual acuity did not have a statistically significant influence on the result. In the type I diabetes groups retinal detachment occurred considerably less often when early vitrectomy was performed. In the type II diabetes groups there was no statistically significant difference in its frequency between early or deferred vitrectomy groups.
The Authors of the DRVS explain the reason why performing early vitrectomy brings benefits in type I diabetes only and not in type II asserting that in type I diabetes the DR has more aggressive characteristics, particularly when the duration of the disease is less than 20 years. The more aggressive progression of neovascularisation, of fibrous proliferation contraction and of vitreoretinal tractions in type I diabetes explain the fact that deferring PPV by 1 year results in worsening of DR with consequent reduced visual acuity recovery after surgery. Other studies have concluded that early vitrectomy for eyes with recent extensive preretinal haemorrhage, retinal traction and tractional detachment [15] and for progressive PDR [16] is preferable even in type II diabetes.

Prompt PPV is also indicated for dense premacular haemorrhage. Another review has demonstrated that patients who received PPV within 1 month from the haemorrhage achieved better final visual acuity. None of these patients developed significant macular traction, while patients who did not receive PPV within 4 weeks all progressed to late macular traction [17].

Further indications for early PPV come from a study that compared early versus deferred PPV for PDR with dense subhyaloid haemorrhage and significant visual acuity loss. The results show an improved anatomic success rate ( $85 \%$ versus $73 \%$ of the deferral group) and a higher rate of reading visual capability $(54 \%$ versus $18 \%$ of the deferral group) thereby suggesting that early PPV is preferable [18].

\section{Conclusions}

At present PPV in the treatment of PDR is indicated for eyes with vitreous haemorrhage preventing laser photocoagulation, severe nonclearing vitreous haemorrhage, subhyaloid and premacular haemorrhage (of at least 1 month duration), tractional retinal detachment involving or threatening the macula, combined tractional and rhegmatogenous retinal detachment, progressive fibrovascular proliferation despite panretinal laser photocoagulation and CSMO. It has been demonstrated that early versus deferred vitrectomy brings clinically significant beneficial effects in case of type I diabetes with a duration of less than 20 years.

\section{References}

1. Fong DS, Aiello LP, Gardner TW, et al. Retinopathy in diabetes. Diabetes Care. 2004;27(suppl):84S-7S.

2. Yam J, Kwok A. Update on the treatment of diabetic retinopathy. Hong Kong Med J. 2007;13(1):46-58.

3. The diabetes Control and Complications Trial Research Group. The effect of intensive treatment of diabetes on the development 
and progression of long-term complications in insulin-dependent diabetes mellitus. N Engl J Med. 1993;329:977-86.

4. UK Prospective Diabetes Study Group. Tight blood pressure control and risk of macrovascular and microvascular complications in type 2 diabetes: UKPDS 38. BMJ. 1998;317:703-13.

5. Schrier RW, Estacio RO, Esler A, Mehler P. Effects of aggressive blood pressure control in normotensive type 2 diabetic patients on albuminuria, retinopathy and strokes. Kidney Int. 2002;61:1086-97.

6. Gupta A, Gupta V, Thapar S, Bhansali A. Lipid-lowering drug atorvastatin as an adjunct in the management of diabetic macular edema. Am J Ophthalmol. 2001;37:675-82.

7. Sen K, Misra A, Kumar A, Pandey RM. Simvastatin retards progression of retinopathy in diabetic patients with hypercholestrerolemia. Diabetes Res Clin Pract. 2002;56:1-11.

8. PKC-DRS2 Group, Aiello LP, Davies MD, Girach A, et al. Effect of ruboxistaurin on visul loss in patients with diabetic retinopathy. Ophthalmology. 2006;113:2221-30.

9. Zein WM, Noureddin BN, Jurdi FA, Schakal A, Bashshur ZF. Panretinal photocoagulation and intravitreal triamcinolone acetonide for the treatment of proliferative diabetic retinopathy with macular edema. Retina. 2006;26:137-42.

10. Bandello F, Polito A, Pognuz DR, Monaco P, Dimastrogiovanni A, Paissios J. Triamcinolone as adjunctive treatment to laser panretinal photocoagulation for proliferative diabetic retinopathy. Arch Ophthalmol. 2006;124:643-50.
11. Avery RL, Pearlman J, Pieramici DJ, et al. Intravitreal bevacizumab (Avastin) in the treatment of proliferative diabetic retinopathy. Ophthalmology. 2006;113(1695):e1-15.

12. Chen E, Park CH. Use of intravitreal bevacizumab as a preoperative adjunct for tractional retinal detachment repair in severe proliferative diabetic retinopathy. Retina. 2006;26:699700 .

13. Ho T, Smiddy WE, Flynn Jr HW. Vitrectomy in the management of diabetic eye disease. Surv Ophthalmol. 1992;37 (3):190-202.

14. The Diabetic Retinopathy Vitrectomy Study Research Group. Early vitrectomy for severe vitreous hemorrhage in diabetic retinopathy. Four-year results of a randomized trial. Arch Ophthalmol. 1990;108:958-64.

15. Li X, Jiang Y, Ye C, Li C. Timing of vitrectomy for proliferative diabetic retinopathy in cases with type II diabetes mellitus. Zhonghua Yan Ke Za Zhi. 1999;35(2):116-8.

16. de Bustros S, Thompson JT, Michels RG, Rice TA. Vitrectomy for progressive diabetic retinopathy. Arch Ophthalmol. 1987;105:196-9.

17. O'Hanley GP, Canny CL. Diabetic dense premacular hemorrhage. A possible indication for prompt vitrectomy. Ophthalmology. 1985;94(4):507-11.

18. Ramsay RC, Knobloch WH, Cantrill HL. Timing of vitrectomy for active proliferative diabetic retinopathy. Ophthalmology. 1986;93(3):283-9. 